\title{
ETUDE SOCIO-ANTHROPOLOGIQUE DE LA GESTION DES RESSOURCES NATURELLES : CAS DU FRONT PIONNIER DE SIDERADOUGOU AU BURKINA FASO
}

\author{
D. J.THIAMOBIGA
}

Institut Polytechnique SHALOM 11 BP 1435 Ouagadougou 11, Burkina Faso. Email : thiamojacques@yahoo.fr

RESUME

Le Burkina Faso est un pays sahélien dont l'économie est essentiellement agricole. Ses ressources naturelles se sont véritablement dégradées au cours de ces dernières décennies. Les causes de cette dégradation sont, entre autres, l'érosion hydrique et éolienne, les changements climatiques, les actions anthropiques. Cette dégradation touchait essentiellement la partie septentrionale voire la partie sahélienne touche. De nos jours, elle touche même les zones occidentales et australes du pays. Au Nord, elle a transformé la flore de cette zone en steppes et tend à favoriser la désertification de cette partie du pays. Les zones occidentales et australes du pays subissent les effets induits de cette dégradation à travers des flux migratoires importants des populations et des troupeaux d'animaux du Nord. C'est dans ce contexte que s'est créé le front pionnier de Sidéradougou. Cette zone regorge de grandes potentialités en ressources naturelles dont la gestion rationnelle peut constituer des atouts majeurs pour son développement durable. Le présent document fait le point des travaux initiés dans cette région depuis 1975.

Mots clés : Ressources naturelles, front pionnier, développement durable, Burkina Faso.

\section{ABSTRACT}

NATURAL RESOURCES MANAGEMENT SOCIO-ANTHROPOLIC STUDY: THE PIONEER FRONT CASE OF SIDERADOUGOU, BURKINAFASO

Burkina Faso is a sahelian country whose economy is essentially based on agriculture. Its natural resources have been deteriorated during the last decencies. This situation has become very dangerous in the North of the country. The populations of this area migrate and are welcome in the southern area which called «new pioneer front». That's the case of Sideradougou area which is characterized by great potentialities in natural resources and particularly pastoralist, sylvicol and agricultural resources, weak demographic density (12,8 hbts/Km²), installation of 1700 families by year, great chapel (30 800 beefs, 31500 sheeps and goats).

Key words : Natural resources, pioneer front, sustainable development, Burkina Faso.

\section{INTRODUCTION}

L'un des défis majeurs auquel l'humanité doit faire face, est sans nul doute, celui de la dégradation de l'environnement et ses corollaires, ainsi que les problèmes des changements climatiques. A cet effet, les Nations Unions ont organisé, en 1992, le sommet de Rio sur la planète-terre qui a abouti à la déclaration de Rio. C'est dire donc que la dégradation de l'environnement, en général et des ressources naturelles, en particulier, constitue, de nos jours, une problématique du développement durable à l'échelle de la planète terre et surtout au niveau des pays sahéliens. Aussi, le Comité (Permanent) Inter-Etats de Lutte contre la Sécheresse dans le Sahel (CILSS) at-il initié, dans les années 95, l'approche «gestion des ressources naturelles».

C'est dans ce contexte que le Burkina Faso, pays situé au cœur de l'Afrique occidentale, enclavé et sahélien (Figure 1), s'est engagé dans cette approche par la création de l'Institut de l'Environnement et des Recherches Agricoles (INERA) qui a créé en son sein le département de la Gestion des ressources naturelles, INERA, 
(1997). Ce département est composé de cinq équipes régionales de gestion des ressources naturelles et des systèmes de production. La dégradation de l'environnement et des ressources naturelles se pose de nos jours avec acuité dans ce pays. Elle le menace dans ses fondements existentiels surtout que la désertification de sa partie septentrionale est amorcée.

Ainsi, l'approche «gestion des ressources naturelles» est devenue, non seulement une nécessité, mais surtout un impératif de la survie de ce pays tant pour les régions agricoles dont les ressources naturelles notamment les terres et la flore se sont fortement dégradées, (Régions agricoles du Centre, du Centre Nord, du Nord et du Sahel) que pour celles qui disposent encore d'un potentiel appréciable en ressources naturelles, (Régions agricoles des HautsBassins, de la Boucle du Mouhoun, du SudOuest et des Cascades).
C'est dans ces régions, notamment dans celles des Hauts-Bassins et des Cascades que se sont créés au cours de la décennie 70 et 80 les fronts pionniers ou les zones de migrations humaines et animales massives, (cas du front pionnier de Sidéradougou).

Ce front pionnier se situe dans la Région des Cascades (Figure 2). L'arrivée et l'installation massives des populations humaines et animales qui se sont effectuées, au cours de ces trois dernières décennies, ont eu des effets dégradants de ses ressources naturelles (terres, eau et flore).

Aussi, convient-il dès maintenant de trouver les voies et moyens capables de l'aider à gérer au mieux ses ressources naturelles. L'une de ces voies est l'approche «gestion des ressources naturelles», qui constitue un enjeu stratégique important pour ce front pionnier. C'est l'objet du présent travail.

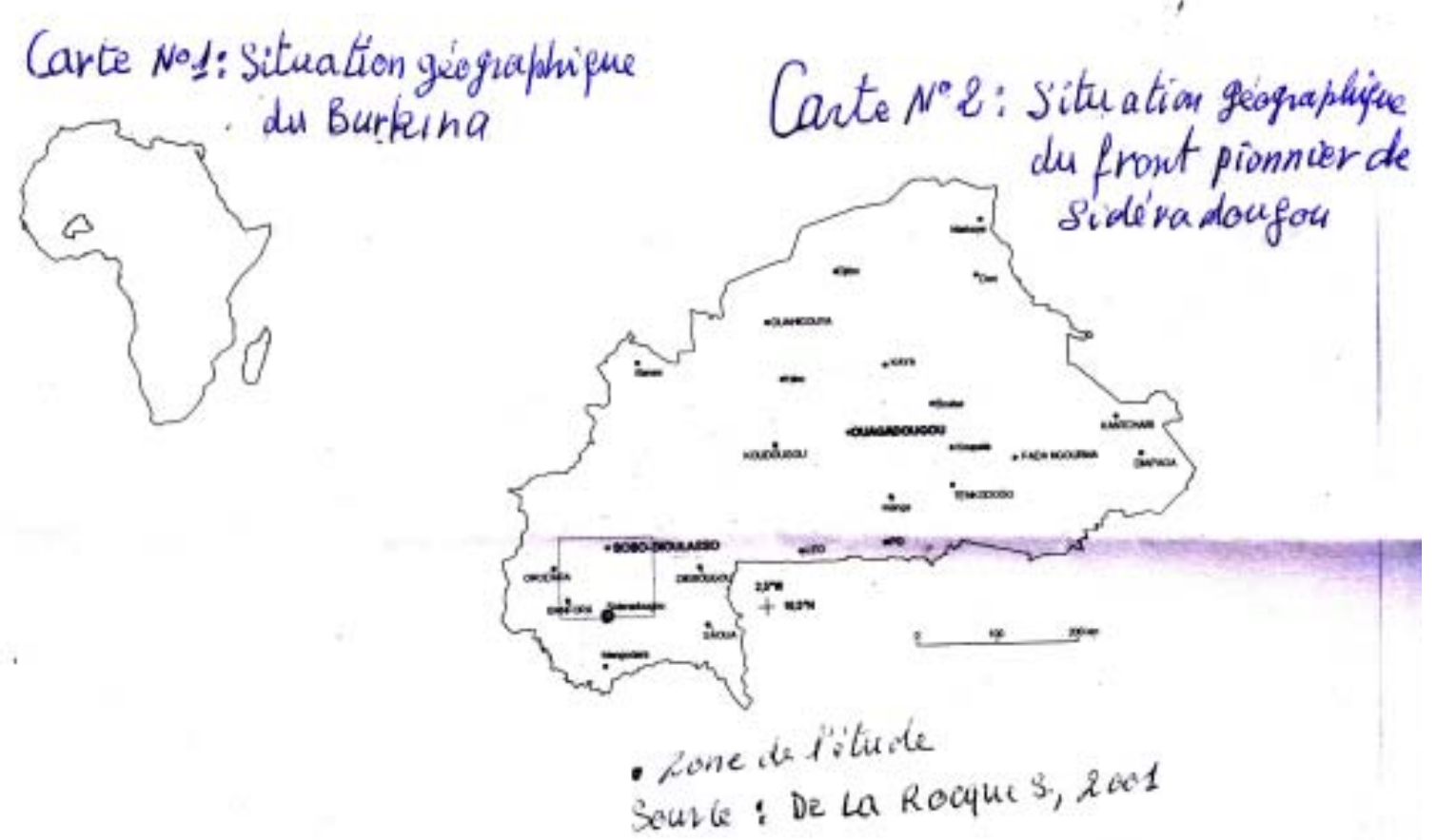

Figures 1 et 2 : Situation géographique du front pionnier. 


\section{MATERIEL ET METHODE}

\section{SITE D'ETUDE}

Le matériel de l'étude porte sur la présentation du front pionnier (site de l'étude) et sur les ressources naturelles. Le front pionnier de Sidéradougou est situé à $85 \mathrm{~km}$ de Banfora, Chef-lieu de la Région des Cascades, (Figure 2). II est aussi appelé la zone agro-pastorale de Sidéradougou.Ce front se situe entre les méridiens $10^{\circ} 32^{\prime}$ et $11^{\circ} 03^{\prime}$ de latitude nord et les parallèles $4^{\circ} 00^{\prime}$ et $4^{\circ} 37^{\prime}$ de longitude Ouest. Au plan administratif, elle est composée de six communes rurales (Tiéfora, Sidéradougou, Mangodara dans la Région des Cascades; Péni, Toussiana, Karrankasso-Vigué et quelques villages de la commune urbaine de BoboDioulasso dans la Région des Hauts-Bassins, (Figure 3).

La situation géographique fait de ce front une des zones les mieux arrosées du pays. La pluviométrie annuelle varie de 900 à $1200 \mathrm{~mm}$. Elle couvre une superficie de 308000 ha subdivisée en quatre sous zones (Soumousso, Péni, Danamandougou et Sidéradougou, Figure 3).

La zone regorge encore de grandes potentialités en matière de ressources naturelles notamment en sols, eau et flore. Les principaux sols rencontrés dans la zone sont les sols ferrugineux lessivés, les sols bruns eutrophes et les sols hydromorphes. Les sols ferrugineux lessivés sont constitués de matériaux d'altération des roches granitiques ou de colluvions sableuses issues des formations gréseuses. Ils présentent un horizon superficiel de couleur grise et ont une texture argilo-sableuse. Ils sont formés d'une cuirasse de latérite, peu propices aux activités agro-sylvo-pastorales. Les sols bruns eutrophes et hydromorphes sont très variés. Ces sols alluvionnaires sont assez fertiles et propices aux activités agro-sylvo-pastorales.

Les principaux cours d'eau sont le Panapra, le Lafigué et le Koba. La pluviométrie annuelle est en moyenne de $1100 \mathrm{~mm}$. La flore est de type sud-soudanien, composée d'un tapis de graminées très dense et des ligneux.
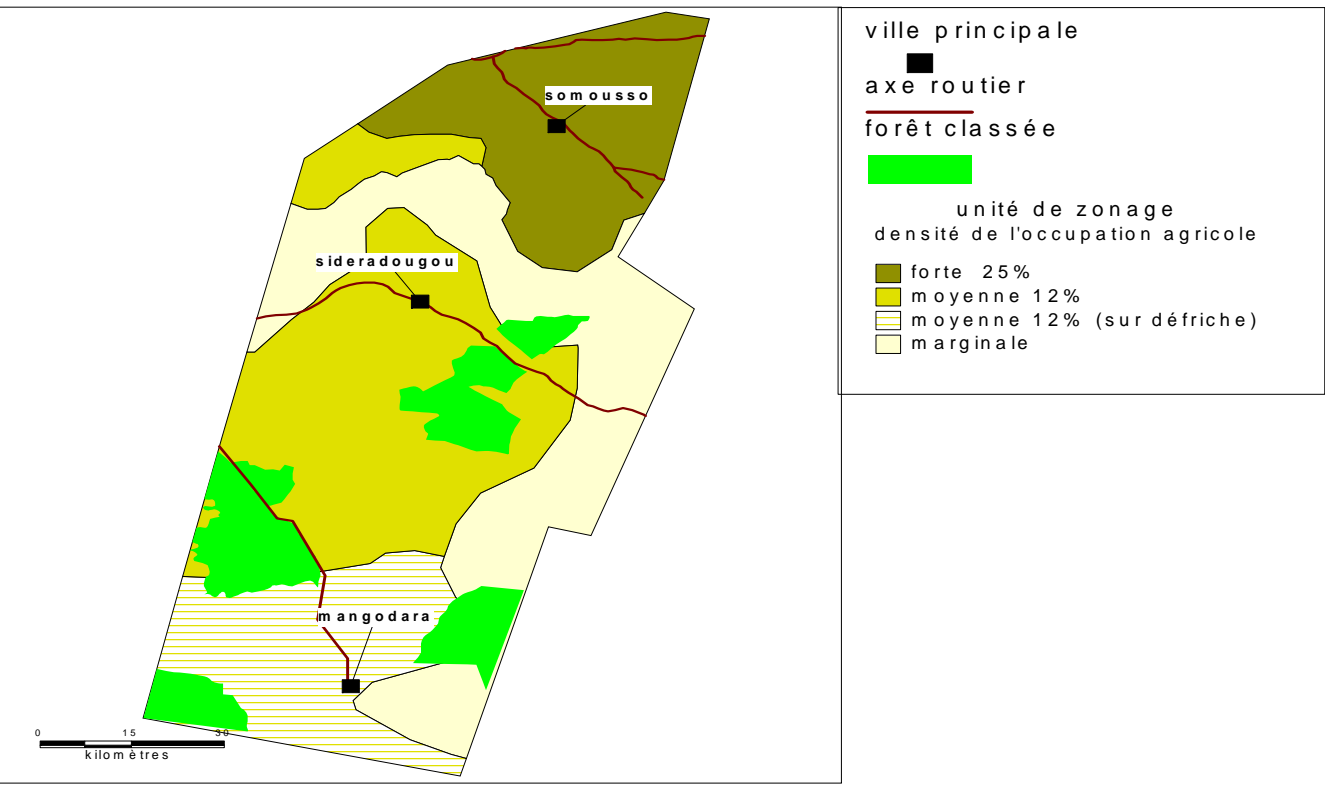

Source : INERA Farako-Ba, Programme GNRSP Ouest

Figure 3 : Présentation globale de la zone. 


\section{METHODES}

Au cours de l'étude, nous avons essentiellement employé les outils de la méthode accélérée de recherche. Les outils employés sont :

- le transect qui permet de faire la carte du village, faisant ressortir toutes les ressources naturelles du village (eau, faune, flore, terres, etc.) ;

- le profil historique qui permet de retracer toute l'histoire du village en mettant l'accent sur les événements les plus importants portant sur la vie du village ;

- le diagramme de VENN qui permet d'analyser les différents éléments qui composent un village et la nature de leurs relations (jeu d'influences réciproques);

- les calendriers qui permettent de programmer les activités dans le temps et l'espace ;

- les interviews semi structurées qui nous ont servi de guides d'entretien.

Ainsi, la collecte des données a porté sur I'histoire des villages qui composent la zone, les caractéristiques de la zone, les modes de migrations, le mode d'accès et d'exploitation des ressources naturelles notamment les ressources agro-pastorales, les types d'activités agro-pastorales dominantes (agriculture, élevage, etc.), le cheptel animal notamment celui des bovins, et la nature des rapports sociaux entre les migrants d'une part et entre les migrants ou allochtones et les autochtones d'autre part.

Outre les travaux réalisés en équipe pluridisciplinaire, nous avons effectué une série d'enquêtes de type exhaustif. Ces enquêtes ont touché à travers les guides d'entretien :

- dix personnes ressources notamment les agents des services déconcentrés (administration, agriculture, environnement et cadre de vie, élevage, etc.) ;

- cinq chercheurs non membres de l'équipe pluridisciplinaire ;

- deux cents producteurs (agriculteurs, éleveurs, sylvicoles, artisans).

L'analyse des données collectées a été faite à l'aide de la méthode hyper empirico dialectique ou la dialectique empirico-réaliste de gurvitch,
(1977) et des méthodes historique, structuraliste, fonctionnaliste et comparative

\section{RESULTATS}

\section{HISTORIQUE DE SIDERADOUGOU}

Historiquement, le village de Sidéradougou (Cheflieu de la zone) a été créé par les dioula venus de Kong de la Côte d'Ivoire et plus précisément par Sidéra. Sidéradougou signifie en Jula le «village de Sidéra». Il aurait décidé de s'installer dans ce village à cause de ses terres propices à l'agriculture. De nos jours, la zone est composée d'une mosaïque de populations qui comportent des groupes autochtones les Dioula (Sidéradougou, Ouara, etc.), les Dogossé (Manaodara), les Tiéfo (Péni), les Bobo, (Koro), les Vigué, (Karranga-Vigué) et les Komono (Ouo). Il est composé de nombreux groupes allochtones dont les plus importants numériquement sont les Mossé (agriculteurs) et les Peuhl, (éleveurs nomades) qui se sont sédentarisés dans la zone.

\section{CARACTERISTIQUES SOCIO-CULTURELLES}

Au niveau administratif, la zone est constituée de cinq communes rurales (Péni, Sidéradougou, Mangodara, Karrankasso-Vigué et Ouo et une partie des villages de la commune urbaine de Bobo-Dioulasso, soit environ 200 villages et campements.

L'évolution spatio-temporelle de la population décrite dans le tableau 1 montre qu'elle a été significative au niveau de trois communes rurales (Sidéradougou, Mangodara et KarrangassoVigué). La population de ces trois (3) communes rurales a évolué de 61295 à 146300 habitants. Les densités des populations varient d'une sous zone à l'autre. II y a des sous zones à faible densité (10 à 15 habitants $/ \mathrm{km}^{2}$ ), à densité moyenne (15 à $30 \mathrm{~h} / \mathrm{km}^{2}$ ) et à forte densité (plus de 30 habitants au $\mathrm{km}^{2}$ ). Les conséquences des flux migratoires sont les pressions agricoles et l'accroissement de la population de la zone. Les pressions agricoles s'établissent comme suit, faibles 10 à $15 \%$, moyennes 15 à $50 \%$ et fortes plus de $50 \%$. A titre d'illustration, l'accroissement de la population du département de Karrangan-Vigué est décrit dans le tableau 1. 
Tableau 1 : Evolution de la population quelques départements étudiés.

\begin{tabular}{lcccccc}
\hline Départ & 1985 & 1996 & $\begin{array}{c}\text { Taux \% par } \\
\text { rapport à 1985 }\end{array}$ & 2006 & $\begin{array}{c}\text { Taux\% par } \\
\text { rapport 1996 }\end{array}$ & $\begin{array}{c}\text { Taux \% par } \\
\text { rapport 1985 }\end{array}$ \\
\hline Mangodara & 16369 & 31986 & 92 & 42083 & 32 & 157 \\
Sidéradougou & 20683 & 31376 & 52 & 41280 & 32 & 100 \\
Karangasso Vigué & 24243 & 47498 & 10 & 62937 & 33 & 157 \\
\hline Total & 61295 & 110860 & 81 & 146300 & 32 & 138 \\
\hline Source : Enquêtes terrain 2006 & & & & &
\end{tabular}

Concernant les systèmes agraires et fonciers, la zone est constituée de terroirs tiéfo, dogossé, komonon et bobo. Les terroirs sont régis par les coutumes de ces groupes. L'accès à la terre se fait par héritage (65\%), par des dons ou cessions volontaires (30\%), des prêts (5\%). Chaque groupe a un chef de terres qui gère les terres. Il existe aussi des formes de co-gestion. C'est le cas du terroir tiéfo sous gestion bobo à Dingasso, sous gestion dioula (Sidéradougou, Dergoué), sous gestion karaboro (Dégué Dégué, Kassandé), du terroir komonon sous gestion Karaboro (Koflandé). Cette co-gestion est établie de manière séculaire et selon des clauses qui empêchent leur remise en cause. De tout ce qui précède, il se dégage que les différents groupes socio-professionnels sont engagés dans la lutte pour la conquête et le contrôle des ressources naturelles.

\section{MODES D'ACCES AUX RESSOURCES NATURELLES}

Les systèmes fonciers et les modes d'accès aux ressources naturelles dans la zone sont à la fois coutumiers et modernes. En effet, bien que le Gouvernement du Burkina Faso se soit doté d'instruments juridiques (textes et lois), il convient de reconnaître l'existence des systèmes agraires et fonciers traditionnels qui, dans les faits quotidiens, régissent la gestion des ressources naturelles. C'est ainsi que chaque groupe autochtone (dioula, bobo, vigué, dogossé, tiéfo) gère son territoire selon les lois coutumières. Bien souvent, les migrants sont obligés d'accomplir des rites d'accès aux terres selon la tradition de chaque groupe autochtone.

Les textes de lois modernes trouvent leurs fondements existentiels dans la Constitution du 2 juin 1991. Citons, entre autres, le code de l'environnement, la loi 006/97 ADP du 31 janvier 1997code forestier du Burkina Faso, fixe l'ensemble des principes fondamentaux relatifs à la gestion des ressources forestières et vise en particulier à établir une articulation harmonieuse entre la nécessaire protection de ces ressources et la satisfaction des besoins des populations.

\section{SYSTEMES DE PRODUCTION}

Les systèmes de production végétale se répartissent du Nord au Sud en trois (3) types, (Figure 3). Au Nord, la zone est cotonnière et maïzicole. Elle se caractérise par des exploitations agricoles équipées et fortement encadrées (groupements villageois, accès au crédit, etc.) par l'intermédiaire des structures et projets de développement. Au Centre, les producteurs pratiquent une agriculture de type vivrier au niveau des campements. La partie méridionale pratique la culture de l'igname en vue de satisfaire aux besoins des marchés locaux et des centres urbains (Banfora, BoboDioulasso, etc.). A ce niveau, il convient de signaler que l'évolution des superficies emblavées est très importante à Sidéragougou et à Mangodara. En effet, les superficies emblavées sont passées respectivement entre 14078 et 79808 ha et 12468 et 19511 ha à Sidéradougou et Mangodara (Tableau 2).

Le système d'élevage est de type extensif, caractérisé par un cheptel important (Tableau 3), et par la mobilité du troupeau à la recherche de fourrage et d'eau. Les effectifs d'animaux se sont accrus au cours des dernières décennies dans chacun de sites dont Mangodara est le plus important. II a enregistré la plus forte évolution de l'effectif du cheptel : $151 \%$. Les taux d'accroissement des effectifs du cheptel 
bovin ont peu varié d'un site à l'autre. Au total, la zone a enregistré, au cours des deux dernières décennies (1985 - 2006), un cheptel de 296915 bovins, soit un taux d'accroissement de $121 \%$, pour un espace non extensible avec des ressources limitées dans le temps et dans l'espace.

La zone abrite des forêts classées, qui constituent d'importantes réserves en ressources agricoles, sylvicoles et pastorales tels que les points d'eau permanents, les pâturages en saison sèche, etc. Ces ressources peuvent servir à l'alimentation du bétail. Par ailleurs, il existe des pratiques traditionnelles d'agro-foresterie à laide d'arbres fruitiers non ligneux tels que le Butyrospermum parkii (Karité), le Parkia biglobosa (Néré), etc. Elle est l'une des parties les mieux arrosées du pays.
En effet, la moyenne pluviométrique est de 1 049,40 mm en 68 jours.

Les systèmes de production se caractérisent au Nord par une amorce de modernisation et d'intensification agricoles. A titre d'illustration, le village de Koro a un taux de $75 \%$ d'exploitations équipées en matériel de culture attelée. Cette partie est surtout caractérisée par le développement de la culture du coton. A Mangodara, l'agriculture vivrière est prépondérante et la production d'igname importante. On y observe également le développement de l'arboriculture fruitière (manguiers, anacardiers) et quelques forêts classées. Par ailleurs, les activités d'élevage prennent de plus en plus de l'ampleur dans la zone, suite aux diverses migrations successives des éleveurs du Nord vers la zone.

Tableau 2 : Evolution des superficies emblavées des départements étudiés.

\begin{tabular}{lccc}
\hline Zones & Superficies (Ha) 1985 & Superficies (Ha), 2006 & Taux \% par rapport à 1986 \\
\hline Sidéradougou & 14078 & 79808 & 467 \\
Karangasso Vigué & Non disponible & 66724 & - \\
Mangodara & 12468 & 19511 & 56 \\
\hline Total & - & 166043 & \\
\hline
\end{tabular}

Source : Enquêtes terrain 2007

Tableau 3 : Evolution des effectifs du cheptel bovin des départements étudiés.

\begin{tabular}{lccc}
\hline Départements & Décembre1986 & Décembre 2006 & $\%$ \\
\hline Mangodara & 18043 & 45671 & 151 \\
Sidéradougou & 83078 & 175919 & 112 \\
Karangasso Vigué & 20106 & 41616 & 107 \\
\hline Total & 121227 & 263206 & 117 \\
\hline
\end{tabular}

Source : Enquêtes terrain 2006

\section{DISCUSSION}

De la présentation des résultats de l'étude, il se dégage des faits anthropologiques majeurs lus dont les plus importants sont les migrations massives, les fortes pressions agricoles sur les ressources naturelles, l'obligation pour tous ceux qui accèdent à la terre de faire des rites agraires, l'évolution significative des superficies emblavées et les effectifs du cheptel.

L'histoire de Sidéradougou montre que son peuplement s'es fait par des vagues successives allant de l'arrivée des groupes autochtones (les Dioula, les Tiéfo, les Dogossé, les Konon) à celle des Mossé et des Peulh. La majeure partie des enquêtés (95\%) soutient que ces populations 
autochtones et allochtones se sont installées dans la zone à la recherche des ressources naturelles (terres, eau et flore) dont elle regorge.

Si l'arrivée des populations autochtones s'est faite de longue date, celle des allochtones est assez récente. Elle est conséquence aux sécheresses des années 1975 et 1980 survenues au Plateau central et au Nord du pays. C'est ainsi que les Mossé du Plateau central et du Nord ont migré dans la zone. Les Mossé sont des grands cultivateurs qui, pour la plupart, ne pouvaient rester dans les zones aux sols fortement dégradés. D'ailleurs, ces zones sont les plus peuplées du Burkina Faso.

Quant aux Peuhl, ils sont des peuples nomades qui étaient dans la majorité dans la zone septentrionale du pays. Cette zone s'est forte dégradée suite aux sécheresses successives, rendant ainsi très difficile l'élevage notamment l'élevage bovin. D'ailleurs, les sécheresses ont décimaient une grande partie des effectifs du cheptel. Ce sont là autant des raisons qui justifient les migrations massives des Peuhl et de leurs troupeaux dans la zone.

Notons aussi que la zone a été autrefois peu propice à l'installation massive des populations à cause de la mouche tsé-tsé, agent vecteur des trypanosomiases. Par ses travaux, le Centre International des Recherche-Développement sur l'Elevage en zone Subhumide (1983) a contribué à libérer la zone des glossines, agents vecteurs des trypanosomiases. C'est dans ce cadre que nous situons les travaux de Meallet, (1997) et d'Ouédraogo (2002). En somme, la zone regorge de nombreuses potentialités en matière de ressources naturelles. C'est le cas des principales ressources agro-sylvo-pastorales (les terres, l'eau et la flore. Elle constitue de ce fait une zone d'accueil des populations humaines et animales qui migrent des autres parties du pays. Elles viennent s'y installer. Ce qui a posé nécessairement des problèmes de sédentarisation dont parle Diallo, (1987).

Enfin, il convient de noter que les Mossé sont les plus nombreux non seulement de la zone mais du pays. Généralement, les populations autochtones de la zone sont des petits groupes ethniques. C'est l'un des constats majeurs que nous avons faits lors de nos les travaux, Thiamobiga (1975, 2002, 1995, 2009). D'ailleurs, l'histoire de la zone est intimement liée à l'arrivée et à l'installation de chaque groupe ethnique qui la compose. A titre d'illustration, l'histoire de Sidéradougou est liée à celle des Dioula venus de Kong de la Côte d'Ivoire. De même, ces travaux indiquent que la zone est peuplée par une mosaïque de groupes autochtones (dioula, tiéfo, dogossé, bobo, etc.) et de groupes allochtones issus des migrations (Mossé, Peuhl, etc.). Les différents groupes ethniques se sont installés dans la zone à cause de sa richesse en ressources naturelles notamment pour son gros potentiel agro-sylvo-pastoral. C'est ainsi que Lhoste (1989) soutient que les migrants mossi et peulh se seraient installés dans la zone dans les années 1989. II va de soi que ce peuplement de la zone a contribué à modifier l'organisation socio-économique de la zone (Requier-Desjardins, 1995).

Par ailleurs, les études de Liéhoun et al. (1997) de Lalba et al. (1997) d'Ouédraogo et al. (1997) d'Auguisseau et al. (1998) montrent que la zone regorge d'énormes potentialités agro-sylvopastorales. Ainsi, pour sahelconsult, les sols de la zone qui sont composés dans leur grande partie par des sols bruns eutrophes et des sols hydromorphes sont propices aux activités agrosylvo-pastorales. La flore, quant elle, est formée des savanes arbustives, Toutain et al. (1978). Elle est l'une des plus denses du pays, De la Rocque et al. (2001). Elle est formée d'un tapis de graminées très dense et par des ligneux dominés par les Butyrospermum paradoxum (karité) et des Terminalia laxiflora (badamiers), etc. A ce niveau, les forêts classées constituent un gros potentiel en ressources forestières. La zone abrite huit forêts classées dont la superficie totale est estimée à 315500 hectares, Ministère de l'Environnement et du Tourisme, (1998).

Les migrations massives humaines et animales ont expliquent les fortes densités des populations humaines et animales de la zone et leurs corollaires les fortes pressions sur les agrosylvo-pastorales comme l'indiquent les tableaux 1,2 et 3 . La zone avait une population de 55000 habitants, (I'Institut National de la Statistique et de la Démographie, 1985). Pour Clanet et Somé (1982), elle a connu une forte croissance démographique en 16 ans. C'est au cours de la décennie (1985-1995) que les migrations animales ont connu une évolution significative. Ce qui est une des conséquences de la réduction de la fréquence des trypanosomiases et les autres moyens de lutte contre les maladies des animaux (CIRDES, 1983). 
S'agissant des différents modes de gestion des ressources naturelles, il convient de noter que les populations autochtones et allochtones doivent accomplir des rites d'occupation et d'exploitation des terres. Ces rites varient d'un groupe ethnique autochtone à l'autre. Ils se résument pour l'essentiel à l'apport du dolo, des poules aux chefs des terres qui font des sacrifices aux ancêtres. Chez les Bobo (population autochtone qui occupent la partie septentrionale de la zone, ces rites s'inscrivent dans la nature des rapports qui existent entre la nature et les hommes. Pour Ciré Ba I le fondement de ces liens peut s'expliquer par le fait suivant : «l'ancêtre chasseur quitta le village Mandé poursuivant le gibier... II aurait apporté des dieux trouvés dans la savane, montagne, rivière qu'il aurait reçus de Longo (le dieu de la brousse) d'un génie ...».

C'est dans cette optique Sanon (1986) a établi «un pacte entre la nature et l'homme». La nature est le sanctuaire du Do qui ne saurait être occupé sans l'autorisation de celui-ci par le truchement du Longo. Les rites d'occupation et d'exploitation des terres constituent la demande d'autorisation d'occuper la nature que l'homme adresse au Do. Aucun homme, qu'il soit pratiquant de la religion du Do ou non, ne saurait transgresser de manière impunie cette coutume. Ces rites constituent en réalité le mode juridico religieux d'appropriation des terres, car ce que le Do a autorisé aucun Madaré ne peut le remettre en cause au risque d'être châtié par celui-ci.

D'ailleurs, le mode traditionnel d'accès aux ressources naturelles notamment aux terres est bâti autour de l'héritage, des dons qui tendent à être remplacés de nos jours par des ventes des terres. Cette vente n'est conforme ni aux coutumes, ni aux textes des lois modernes qui définissent le cadre juridique d'accès aux ressources naturelles. C'est le cas de la Réorganisation Agraire et Foncière adoptée en 1986 et révisée en 1991 qui font des terres du pays une propriété de l'Etat.

Les différentes analyses que nous venons de faire nous permettent de faire quelques suggestions relatives à une exploitation des atouts, à la protection des forêts classées, à la gestion des conflits et à la levée et à défaut à l'atténuation des contraintes.

La gestion des forêts classées est essentiellement assurée au Burkina par l'Etat. Or, cette gestion ne peut être efficace que si les populations riveraines de ces forêts classées sont fortement impliquées dans cette gestion. C'est l'une des recommandations majeures du congrès forestier mondial tenu à Paris en France en 1991.

C'est aussi le cas du sommet de la terre tenue à Rio-de-Janeiro au Brésil en 1992, sur l'environnement et le développement, a mis un accent tout à fait particulier sur l'urgence et la nécessité d'impliquer les populations rurales comme acteurs à part entière dans la planification et la gestion durable des ressources naturelles. A cet effet, il a défini une approche mondiale visant à mettre fin à la détérioration de l'environnement ou tout au moins à l'atténuer. Cette approche vise à susciter et à encourager les efforts nationaux et internationaux à rechercher un développement durable et écologiquement rationnel.

Le principe 9 définit ainsi l'itinéraire scientifique de la diffusion des technologies. Quant au principe 22, il détermine les rôles des communautés et des Etats dans l'édification du développement durable. II stipule que : «Les populations et les communautés autochtones et les autres collectivités locales ont un rôle vital à jouer dans la gestion de l'environnement et le développement du fait de leurs connaissances du milieu et leurs pratiques traditionnelles. Les Etats devraient reconnaître leur identité, leur culture et leurs intérêts, leur accorder tout l'appui nécessaire et leur permettre de participer efficacement à la réalisation d'un développement durable, (action 21, 1993).

Ces deux principes sont similaires et/ou complémentaires à ceux de la thèse que nous avons élaborée et que nous défendons. Ils se résument comme suit «il ne peut avoir de développement durable et véritable d'une société s'il ne s'enracine à sa culture et s'il n'est marqué du sceau de son identité». Partant de ces principes, nous pouvons affirmer en guise d'hypothèse de recherche que la protection et la préservation de la nature ou de l'environnement ne peuvent réussir véritablement au Burkina que si elles s'enracinent à la culture et à l'identité de nos sociétés. Car elles ont des dimensions socio-culturelles et par conséquent des dimensions identitaires.

II va de soi que l'intensification agricole ne peut se faire sans un équipement approprié. Aussi, convient-il d'aider les producteurs notamment ceux du coton et de l'igname à s'équiper en matériel agricole approprié (charrues, bœufs et éventuellement tracteurs). 
Au niveau de l'élevage, les mêmes actions peuvent être menées en fonction des spéculations. Cela pourra aboutir à l'intensification de l'élevage à travers la mise à disposition des éleveurs, des intrants zootechniques. Cette intensification aboutira à long terme à une véritable sédentarisation des éleveurs.

\section{CONCLUSION}

Les différentes analyses montrent que la zone de Sidéradougou regorge de nombreuses potentialités en matière de ressources naturelles (terres, eau, flore, etc.). Elle dispose ainsi de nombreux atouts dans ce domaine. Force a été de constater que la gestion des ressources naturelles rencontre de nombreuses contraintes (migrations spontanées, cultures consommatrices de grands espaces agricoles (coton et igname), élevage extensif, conflits sociaux, etc.

Au-delà de ces contraintes, il convient de dire que la gestion des ressources naturelles constitue un ensemble d'enjeux stratégiques pour le développement durable de la zone. Elle peut par conséquent réaliser son développement durable par une gestion plus rationnelle de ses ressources naturelles. Nous avons fait quelques suggestions aux décideurs politiques du pays susceptibles de les aider à gérer au mieux ses ressources naturelles.

\section{REFERENCES}

Auguisseau X. et A. Paré. 1998. Caractérisation régionale de l'emprise agricole et de sa dynamique dans une zone de migration, Communication faite au colloque sur l'élevage, Ouagadougou, p. 6

Centre International de Recherche-Développement sur l'Elevage en zone Subhumide. 1983. Projet de lutte intégrée contre les glossines dans la zone de Sidéradougou, Bilan d'une première année de lutte par association d'écrans imprégnés d'insecticides et lâchers de mâles, CIRDES, BoboDioulasso Burkina Faso, $16 \mathrm{p}$

Clant J. C. et P. H. Somé. 1983. Réactualisation de l'étude de la zone pastorale d'accueil de Sideradougou, $43 \mathrm{p}$.

De la Rocque S. 2002. Lutte antivectorielle ciblée aux points épidémiologiquement dangereux de la zone agropastorale de Sidéra- dougou, Rapport d'étape à l'issue de la première année d'intervention $34 \mathrm{p}$.

Diallo H. 1987. Problématique de la sédentarisation de l'élevage au Burkina Faso. (Situation- Contraintes- Perspectives). Mémoire Ingénieur, Institut polytechnique Rural de Katibougou, 148 p.

Duffumier M. 1994. Conditions à promouvoir pour la viabilité des systèmes de productions agricoles durables dans les pays d'Afrique sahélienne et soudanienne, Dakar, p. 8.

Gurvtich G. 1977. Dialectique et Sociologie PUF Paris $307 \mathrm{p}$.

Institut National de la Statistique et de la Démographie. 1985. Recensement général de la population, INSD Ouagadougou p. 55.

Lalba A., Liéhoun E., Thiamobiga D. J. et S. Ouédraogo. 1997. Les enjeux des modes de conduite des troupeaux pour une gestion rationnelle des ressources naturelles de la zone de Sidéradougou. Communication faite au colloque sur l'élevage, Ouagadougou, $13 \mathrm{p}$.

Liéhoun E., Ouédraogo S., Thiamobiga D. J. et A. Lalba. 1997, Elevage et gestion des terroirs dans le sud - ouest du Burkina Faso. Colloque sur l'élevage au Burkina Faso, Ouagadougou, $12 \mathrm{p}$.

Lhoste P. 1999. Actes du séminaire sur l'élevage en zone cotonnière, études et synthèses de l'IEMVT, 36 CIRAD-IEMVT, Maison Alfort, France/CEBV, Ouagadougou Burkina Faso 25 - 28 octobre, $340 \mathrm{p}$.

Lhoste P. 1993. Zootechnie des régions chaudes, les sytèmes d'élevage, Ministère de coopération CIRAD, $288 \mathrm{p}$

Meallet C. 1997. Recensement et cartographie du cheptel bovin de la zone de Sidéradougou, Mémoire de DESS, Production animale des régions chaudes, Ecole nationale de vétérinaire d'Alfort, $51 \mathrm{p}$.

Ministère de l'Environnement et du Tourisme. 1998. Etat récapitulatif des parcs nationaux, des ressources des Faunes et Forêts classées, Rapport technique, p. 7.

Ministère des Ressources Animales. 2001. Les statistiques du secteur d'élevage au Burkina Faso, Année 1999, Ouagadougou Burkina Faso, 86 p.

Ouédraogo A. 2002. Durabilité de la lutte contre le trypanosome animale africaine dans la zone de Sidéradougou, aspects socioéconomiques, Mémoire de fin d'études, 
université Polytechnique de BoboDioulasso, Burkina Faso, 60 p.

Ouédraogo S., Thiamobiga D. J., Lalba A. et E. Liéhoun. 1997. Etude typologique des systèmes d'élevage de la zone agropastorale de Sideradougou, Colloque sur l'élevage, Ouagadougou, p. 5.

Requier-Desjardins M. 1995. Système d'élevage, mobilité des troupeaux et gestion des ressources pastorales en Afrique soudanosahélienne, enquête dans la zone cotonnière du Burkina Faso, Mémoire de DEA, université de paris I panthéon-Sorbonne, $97 \mathrm{p}$

Shaleconsult. 1991. Schéma directeur de mise en valeur de la zone aménagée de Sidéradougou, Rapport de synthèse BoboDioulasso Burkina Faso, SAHELCONSULT Ouagadougou, $61 \mathrm{p}$

Thiamobiga D. J. 1975. Enquêtes sur l'introduction de la culture attelée dans le secteur agricole de Sidéradougou, Rapport de stage Centre Agricole Polyvalent de Matourkou, $37 \mathrm{p}$

Thiamobiga D. J. et M. Dayamba. 2002. Enquêtes socio-économiques dans la zone de Sidéradougou, Rapport de stage, Centre Agricole Polyvalent de Matourkou, $47 \mathrm{p}$

Thiamobiga D. J., Liéhoun E., Ouédraogo S. et A. Lalba. 1997. Les enjeux de la culture attelée pour l'appropriation de l'élevage dans la société madaré du Burkina Faso, article présenté en co-publication au Comité Technique «Elevage» / CNRST / IDR / CIRDES, octobre 1997).

Touré S. M. 1991. Le nomadisme et le pastoralisme face à la sauvegarde du patrimoine naturel, Bruxelles, pp. 5 - 6

Toutain B., Dumas R. et G. Tacher. 1978. Zone pastorale d'accueil de Sidéradougou (Haute Volta). Etude préliminaire. Etude préliminaire CIRAD-IEMVT, Maisons-Alfort, France, $191 \mathrm{p}$. 